\section{Controle da leishmaniose visceral canina por eutanásia: estimativa de efeito baseado em inquérito e modelagem matemática}

\author{
Control of canine visceral leishmaniasis by \\ euthanasia: estimated effect based on a \\ survey and mathematical modeling
}

\section{Control de la leishmaniasis visceral canina por eutanasia: estimación del efecto basado en una encuesta y modelo matemático}

Danielle Nunes Carneiro Castro Costa 1

Cláudia Torres Codeço 2

Patricia Marques Moralejo Bermudi 1

Lilian Aparecida Colebrusco Rodas 3

Cáris Maroni Nunes 4

Roberto Mitsuyoshi Hiramoto 5

José Eduardo Tolezano 5

Francisco Chiaravalloti Neto 1

doi: 10.1590/0102-311X00221418

\section{Resumo}

A leishmaniose visceral é uma doença emergente e negligenciada em processo de expansão para áreas urbanas. A incidência da doença humana está relacionada com a infecção canina. Araçatuba e Birigui são municípios do Estado de São Paulo, Brasil, com soroprevalência da infecção canina de 8 a 10\%, que empregam estratégias de controle voltadas ao reservatório canino baseado em inquérito sorológico e eutanásia dos cães soropositivos. Usando dados desses programas de controle para parametrizar modelos matemáticos, este estudo avaliou a eficácia dessas atividades. Estimamos que o controle atualmente empregado é capaz de reduzir em cerca de 20\% a incidência de casos de leishmaniose visceral canina (LVC). Considerando-se um controle contínuo e um esforço das atividades de inquérito sorológico igual ao triplo da média do observado em Araçatuba e Birigui, a atividade de eutanásia de cães com diagnóstico positivo seria efetiva para o controle da infecção canina. Embora teoricamente possível, na prática, o controle da LVC com as estratégias preconizadas atualmente é insuficiente, pois exigiria superpor dificuldades enfrentadas por estas atividades como falta de recursos materiais, humanos e financeiros, além das questões éticas e jurídicas associadas.

Leishmania; Leishmaniose Visceral; Doenças do Cão; Reservatórios de Doenças

\section{Correspondência}

D. N. C. C. Costa

Faculdade de Saúde Pública, Universidade de São Paulo. Av. Dr. Arnaldo 715, São Paulo, SP 01246-904, Brasil. ieovah1@gmail.com

1 Faculdade de Saúde Pública, Universidade de São Paulo, São Paulo, Brasil.

2 Programa de Computação Científica, Fundação Oswaldo Cruz, Rio de Janeiro, Brasil.

3 Serviço Regional 9, Superintendência de Controle de Endemias, Araçatuba, Brasil.

4 Faculdade de Medicina Veterinária de Araçatuba, Universidade Estadual Paulista, Araçatuba, Brasil. 5 Núcleo de Parasitoses Sistêmicas, Instituto Adolfo Lutz, São Paulo, Brasil. 


\section{Introdução}

A leishmaniose visceral (LV) é a forma clínica mais grave das leishmanioses e é considerada uma doença emergente e negligenciada pela Organização Mundial da Saúde (OMS). Até a década de 1990, com exceção da Região Sul, casos da doença eram registrados em todas as regiões brasileiras. Todavia, com a adaptação do vetor a ambientes urbanos, a dispersão da LV parece ter atingido toda a extensão do território brasileiro, com registro do vetor e de casos caninos também no extremo sul do país 1,2. Estudos epidemiológicos em áreas urbanas têm verificado sobreposição entre locais com incidência de casos humanos e elevada soroprevalência canina, evidenciando a estreita relação entre a infecção canina e a humana 3,4 .

Atualmente, a estratégia usada como controle da infecção do reservatório canino na rotina do Programa Nacional de Vigilância e Controle da Leishmaniose Visceral (PNVCLV) é a eutanásia de cães soropositivos. Apesar da larga utilização dessa estratégia, muitos são os desafios na sua operacionalização e manutenção, como infraestrutura inadequada nos municípios, financiamento insuficiente ou inexistente para seu desenvolvimento e, especialmente, as questões éticas envolvidas 5,6,7,8, o que leva a questionamentos quanto à sua efetividade 5,9. Alternativas à eutanásia têm sido pesquisadas, como a vacinação de cães, o tratamento de cães infectados e o uso de coleiras impregnadas com deltametrina 10,11,12,13. Dentre estas estratégias, a última apresenta resultados promissores quanto à sua utilização como medida de saúde pública 14,15. Ainda assim, a eutanásia combinada com a borrifação de domicílios, constitui a principal linha de ação implementada pelo Ministério da Saúde no Brasil 1.

Araçatuba e Birigui foram os primeiros municípios do Estado de São Paulo a confirmarem casos autóctones da LV em 1999 e, desde então, estes municípios tornaram-se endêmicos para a doença 16 .

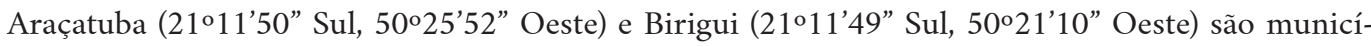
pios adjacentes e com características socioepidemiológicas semelhantes. Estão localizados na região noroeste do Estado de São Paulo e são as principais cidades da região administrativa de Araçatuba. A região tem temperatura média anual de $23^{\circ} \mathrm{C}$ e precipitação acumulada anual de $1.229,5 \mathrm{~mm}$. Araçatuba tem uma população estimada em 194.874 habitantes e $52 \mathrm{~km}^{2}$ de área urbana (Instituto Brasileiro de Geografia e Estatística. https://cidades.ibge.gov.br/v4/brasil/sp/aracatuba/panorama, acessado em 20/Nov/2018). Birigui é um município menor, com população estimada em 120.692 habitantes e $46 \mathrm{~km}^{2}$ de área urbana (Instituto Brasileiro de Geografia e Estatística. https://cidades.ibge.gov.br/ brasil/sp/birigui/panorama, acessado em 20/Nov/2018).

Segundo dados da Superintendência de Controle de Endemias do Estado de São Paulo (SUCEN), no período de 2012 a 2014 a incidência dos casos humanos de LV foi de 3,4 por 100 mil habitantes em Araçatuba, e 7,3 por 100 mil habitantes em Birigui. Esses valores representam uma drástica redução em relação aos registrados de cinco anos antes, de 20,0 casos por 100 mil habitantes em Araçatuba ${ }^{4}$ e 14,0 casos por 100 mil habitantes em Birigui 16. Nesse mesmo período foi registrada, em Araçatuba, redução de soroprevalência da infecção canina de 18 para 8\% 4. Quanto a Birigui, a soroprevalência no período foi de 10\%, segundo dados coletados junta à SUCEN, mas não há informação relativa aos cinco anos anteriores, uma vez que não houve inquérito sorológico em anos anteriores a 2010. As ações de controle da leishmaniose visceral canina (LVC) nesses municípios seguem as diretrizes do PNVCLV.

Considerando-se a importância histórica e epidemiológica de Araçatuba e Birigui para a disseminação da LV no Estado de São Paulo, o objetivo deste estudo foi parametrizar modelos matemáticos para representar os cenários epidemiológicos desses municípios e estimar o efeito do esforço de eutanásia de cães soropositivos como estratégia de controle da LVC. A modelagem matemática permite responder às seguintes perguntas: Qual é o impacto, em redução de LVC, do esforço atual de controle realizado por esses municípios? Quão maior seria o esforço necessário para alcançar a meta de eliminação da LVC? A modelagem permite colocar em contexto o uso da eutanásia como estratégia e ponderá-la em relação às alternativas disponíveis? 


\section{Metodologia}

A dinâmica da transmissão da LVC em Birigui e Araçatuba foi representada por modelos matemáticos dinâmicos. A literatura tem diversas propostas de modelos para LVC, variando no número de compartimentos e nas taxas entre estes 17,18,19,20. Essas variações refletem a existência de lacunas no conhecimento sobre a história natural da LVC, com poucos estudos disponíveis para basear a elaboração dos modelos 21,22,23. Aqui, o modelo de Costa et al. $24\left(\mathrm{SEI}_{2} \mathrm{D}\right)$ foi utilizado, o qual contém seis compartimentos que representam a história da infecção na população canina (caixas pretas na Figura 1). Nesse modelo não há representação explícita da população de insetos vetores. Além desse modelo, foram exploradas duas variantes: a primeira inclui três compartimentos para representar a população de flebotomíneos $\left(\mathrm{SEI}_{2} \mathrm{DV}\right)$, como em Shimozako et al. 20 (caixas azuis na Figura 1), e a segunda variante inclui a recuperação espontânea de uma fração dos cães com infecção assintomática ( $\mathrm{SEI}_{2} \mathrm{DR}$ ), como em Sevá et al. 19 (caixa verde na Figura 1). Com esses modelos alternativos, foi possível estudar a sensibilidade das conclusões encontradas aos pressupostos que fundamentam a construção do modelo original.

\section{Figura 1}

Diagramas dos modelos de dinâmica populacional de leishmaniose visceral canina (LVC).

Cães

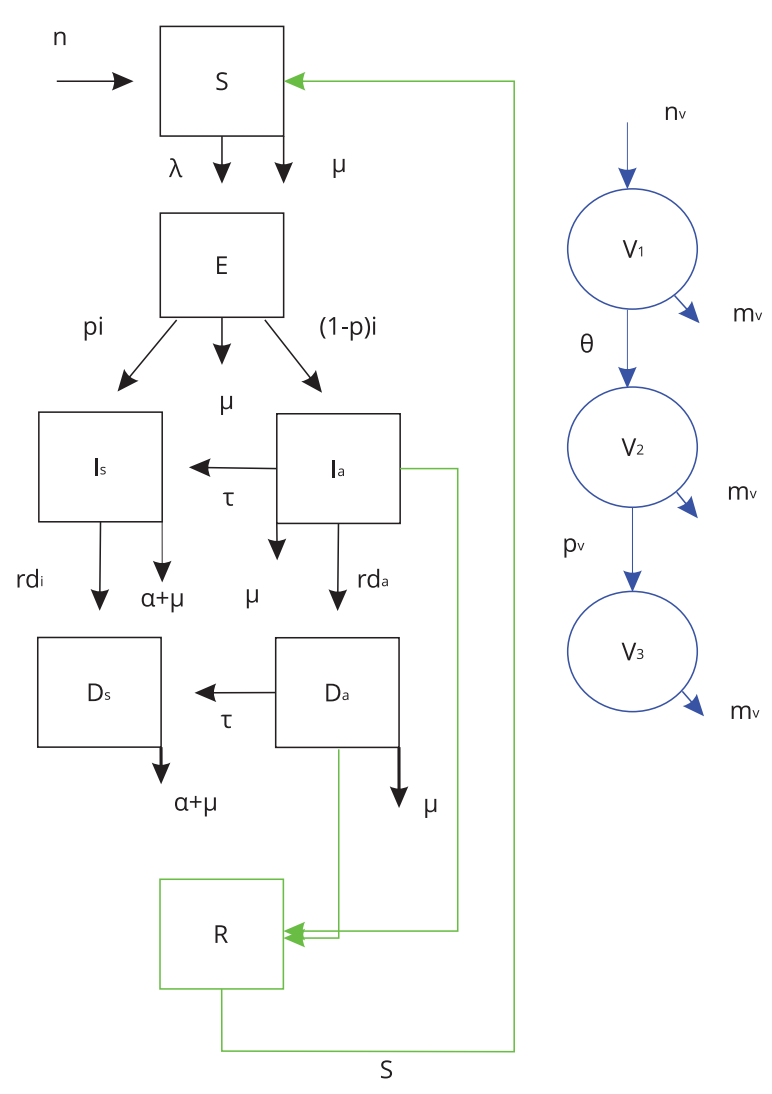

Nota: os símbolos são descritos no texto e na Tabela 1. O modelo de LVC proposto por Costa et al. 24 (SEI 2 D) contém apenas os compartimentos caninos (fluxograma da esquerda com linhas pretas). A versão aumentada do modelo de LVC inclui os compartimentos da população de vetores (fluxograma em azul) e é chamado de $\mathrm{SEI}_{2} \mathrm{DV}$. O modelo com classe de recuperados inclui o compartimento em verde, chamado de $\mathrm{SEI}_{2} \mathrm{DR}$. 
Em todos os modelos, a população de cães (e de flebotomíneos, quando pertinente) foi mantida constante ao longo do tempo, ou seja, a taxa de natalidade (entrada de indivíduos na população) correspondeu à taxa de mortalidade, fossem por causas naturais ou por efeito da doença ou do controle. Uma das dificuldades do controle da LVC é o hábito de muitos donos de cães de adotar um novo animal após a eutanásia dos soropositivos 25 . Dessa forma, assume-se que na presença de controle por eutanásia, 100\% dos cães eliminados são repostos na população por adoção.

\section{Modelo $\mathrm{SEI}_{2} \mathrm{D}$ (de Costa et al.)}

A dinâmica da LVC proposta por Costa et al. 24 é descrita por seis equações cujos parâmetros estão descritos na Tabela 1.

$$
\begin{gathered}
\frac{d S}{d t}=n-\lambda(t) S-\mu S \\
\frac{d E}{d t}=\lambda(t) S-(i+\mu) E \\
\frac{d I_{s}}{d t}=p i E+\tau_{a s} I_{a}-r d_{i} I_{s}-(\alpha+\mu) I_{s} \\
\frac{d I_{a}}{d t}=(1-p) i E-\tau_{a s} I_{a}-r d_{a} I_{a}-\mu I_{a} \\
\frac{d D_{s}}{d t}=r d_{i} I_{s}+\tau_{a s} D_{a}-(f+\alpha+\mu) D_{s} \\
\frac{d D_{a}}{d t}=r d_{a} I_{a}-\tau_{a s} D_{a}-(f+\mu) D_{a}
\end{gathered}
$$

Nesse modelo, cães suscetíveis $(S)$, ao se infectarem a uma taxa $\lambda(t)$, entram em estado de latência (E) durante o qual não participam da transmissão da infecção e ainda são negativos para os testes sorológicos. Após o estado de latência, que dura em média um ano 26, os cães tornam-se infectantes, sendo que uma fração $p$ destes tem para um quadro sintomático $\left(I_{s}\right)$ e uma fração $(1-p)$ evolui para infecção assintomática $\left(I_{a}\right)$. Estudos sugerem que uma parcela dos cães assintomáticos pode evoluir sinais clínicos da doença mais tardiamente: este processo foi incorporado ao modelo por meio da taxa $\tau_{a s}$ de saída do compartimento de assintomático para sintomático 27,28. O modelo não tem uma classe de recuperados, isto é, assume-se que não há cura espontânea para LVC. Além disso, não tem uma representação explícita do vetor, de forma que o contato modelado é direto entre cães suscetíveis e infectados. A expressão para a força de infecção, nesse caso, tem como parâmetros a taxa de transmissão $(\beta)$ e a probabilidade de transmissão por contato com animal sintomático $\left(q_{s}\right)$ ou assintomático $\left(q_{a}\right)$ :

$$
\lambda(t)=\beta / N\left(q_{a}\left(I_{a}+D_{a}\right)+q_{s}\left(I_{s}+D_{s}\right)\right)
$$

A aplicação do controle por eutanásia dos cães soropositivos é representada por dois compartimentos: $D_{s}$ representa animais sintomáticos com diagnóstico positivo e $D_{a}$ representa animais assintomáticos com diagnóstico positivo. A entrada dos cães nesses compartimentos depende da cobertura de inquérito $(r)$, que corresponde à taxa de busca e coleta de amostra de sangue dos cães e da sensibilidade do teste diagnóstico para cães sintomáticos $\left(d_{i}\right)$ e assintomáticos $\left(d_{a}\right)$. A taxa de saída desses compartimentos depende da taxa de realização de eutanásia $f$, além da morte natural ou por doença.

\section{Modelo com vetores $\left(\mathrm{SEI}_{2} \mathrm{DV}\right)$}

No modelo de LVC com vetor, aos compartimentos do modelo $\mathrm{SEI}_{2} \mathrm{D}$ foram acrescidas três equações para descrever a dinâmica da infecção na população de flebotomíneos utilizando-se como base o modelo de transmissão vetorial descrito em Nishiura 29. O modelo é ilustrado na Figura 1 e inclui os compartimentos em preto e azul. A população de vetores é dividida em suscetíveis $\left(V_{1}\right)$, latentes $\left(V_{2}\right)$ e 


\section{Tabela 1}

Parâmetros e símbolos utilizados nos modelos matemáticos de dinâmica de leishmaniose visceral canina.

\begin{tabular}{|c|c|c|c|c|}
\hline Símbolo & Significado & Valor Araçatuba & Valor Birigui & Referência \\
\hline$S$ & Número de cães suscetíveis & 7.829 & 11.397 & Valores obtidos após calibração do modelo de \\
\hline E & Número de cães com infecção latente & 19 & 15 & Costa et al. 24, no equilíbrio endêmico \\
\hline $\mathrm{l}_{\mathrm{a}}$ & Número de cães com infecção assintomática & 424 & 342 & \\
\hline$I_{s}$ & Número de cães com infecção sintomática & 220 & 179 & \\
\hline $\mathrm{D}_{\mathrm{a}}$ & $\begin{array}{l}\text { Número de cães assintomáticos diagnosticados } \\
\text { positivos }\end{array}$ & 22 & 12 & \\
\hline$D_{s}$ & $\begin{array}{l}\text { Número de cães sintomáticos diagnosticados } \\
\text { positivos }\end{array}$ & 21 & 11 & \\
\hline N & População canina total & 8.538 & 11.958 & IBGE, 2010 * \\
\hline$\mu$ & Taxa de mortalidade natural & \multicolumn{2}{|c|}{$1 / 4.385 \mathrm{dia}^{-1}$} & Shimozako et al. 20, 2017 \\
\hline A & Taxa de mortalidade pela infecção & \multicolumn{2}{|c|}{$1 / 552$ dia-1 } & Shimozako et al. 20, 2017 \\
\hline$\tau_{\text {as }}$ & $\begin{array}{c}\text { Taxa de progressão de cães assintomáticos } \\
\text { para sintomáticos }\end{array}$ & \multicolumn{2}{|c|}{$1 / 144$ dia-1 $^{-1}$} & Pozio et al. 27, 1981 \\
\hline i & 1/período de incubação intrínseca & \multicolumn{2}{|c|}{$1 / 30$ dia $^{-1}$} & Maia et al. 26, 2010 \\
\hline$\beta$ & Taxa de transmissão no modelo sem vetores & $3,06 \times 10^{-3} \mathrm{dia}^{-1}$ & $2,95 \times 10^{-3} \mathrm{dia}^{-1}$ & Valores encontrados na calibração do modelo \\
\hline $\mathrm{p}$ & $\begin{array}{l}\text { Proporção de cães que evoluíram para } \\
\text { sintomáticos após latência }\end{array}$ & \multicolumn{2}{|c|}{0,32} & Sevá et al. 19, 2016 \\
\hline$q_{a}$ & Infecciosidade de $\mathrm{I}_{\mathrm{a}}$ & \multicolumn{2}{|c|}{0,385} & Laurenti et al. 36, 2013 \\
\hline$q_{s}$ & Infecciosidade de $I_{s}$ & \multicolumn{2}{|c|}{0,247} & Laurenti et al. 36, 2013 \\
\hline r & Cobertura anual de inquérito & 0,088 & 0,075 & $\begin{array}{c}\text { SUCEN } \\
\text { Cenário sem controle }=0 \\
\text { Cenário aumentado }=0,25\end{array}$ \\
\hline$d_{a}$ & Sensibilidade do diagnóstico de assintomáticos & \multicolumn{2}{|c|}{0,47} & Grimaldi et al. 37, 2012 \\
\hline$d_{i}$ & Sensibilidade do diagnóstico sintomáticos & \multicolumn{2}{|c|}{0,98} & Grimaldi et al. 37, 2012 \\
\hline$f$ & Cobertura anual de eutanásia canina & 0,432 & 0,659 & $\begin{array}{l}\text { Cenário sem controle }=0 \\
\text { Cenário aumentado }=0,5\end{array}$ \\
\hline $\mathrm{V}_{1}$ & Número de flebotomíneos suscetíveis & 13.969 & 21.051 & Valores obtidos após calibração do modelo com \\
\hline$v_{2}$ & $\begin{array}{l}\text { Número de flebotomíneos em estágio de } \\
\text { latência }\end{array}$ & 370 & 324 & $\begin{array}{l}\text { vetores, no equilíbrio endêmico. } \\
\text { Esses compartimentos estão presentes no }\end{array}$ \\
\hline$v_{3}$ & Número de flebotomíneos transmissores & 1.057 & 926 & modelo $\mathrm{SEI}_{2} \mathrm{DV}$ \\
\hline a & Taxa média de picadas & \multicolumn{2}{|c|}{0,2 dia $^{-1}$} & Shimozako et al. 20, 2017 \\
\hline$P_{v}$ & 1/período de incubação extrínseca & \multicolumn{2}{|c|}{$1 / 7 \mathrm{dia}^{-1}$} & Courtenay et al. 40, 2017 \\
\hline$m_{v}$ & Taxa de mortalidade do vetor & \multicolumn{2}{|c|}{0,05 dia-1 } & Shimozako et al. 20, 2017 \\
\hline Y & Taxa de recuperação espontânea da infecção & \multicolumn{2}{|c|}{$0,01 \times 1 / 180$ dia-1 $^{1}$} & $\begin{array}{l}\text { Oliva et al. 39, } 2006 \\
\text { 0,01 é a probabilidade de recuperar e 1/180 é a } \\
\text { taxa de recuperação }\end{array}$ \\
\hline s & Taxa de perda de imunidade & \multicolumn{2}{|c|}{$1 / 365 \mathrm{dia}^{-1}$} & Sevá et al. 19, 2016 \\
\hline
\end{tabular}

IBGE: Instituto Brasileiro de Geografia e Estatística; SUCEN: Superintendência de Controle de Endemias do Estado de São Paulo.

* https://cidades.ibge.gov.br/v4/brasil/sp/aracatuba/panorama; https://cidades.ibge.gov.br/brasil/sp/birigui/panorama (acessado em 20/Nov/2018). 
infectantes $\left(V_{3}\right)$, sendo os últimos responsáveis pela transmissão. Ao modelo original de Costa et al. 24 foram acrescidas as equações:

$$
\begin{gathered}
\frac{d V_{1}}{d t}=n_{v}-\theta(t) V_{1}-m_{v} V_{1} \\
\frac{d V_{2}}{d t}=\theta(t) V_{1}-\left(p_{v}+m_{v}\right) V_{2} \\
\frac{d V_{3}}{d t}=p_{v} V_{2}-m_{v} V_{3}
\end{gathered}
$$

sendo que a força de infecção em vetores, $\theta(t)$, depende do contato de vetores suscetíveis com cães infectados, descrito pela expressão:

$$
\theta(t)=a / N\left(q_{s} I_{s}+q_{a} I_{a}\right)
$$

Nesse modelo com vetores, a força de infecção na população canina, $\lambda(t)$, é redefinida de forma a indicar $V_{3}$ como fonte de infecção:

$$
\left.\lambda(t)=\beta_{a} / N\right) V_{3}
$$

\section{Modelo com classe de recuperados $\left(\mathrm{SEI}_{2} \mathrm{DR}\right)$}

Foi investigada também uma variação do modelo original em que uma fração de $10 \%$ dos infectados assintomáticos $\left(I_{a}\right)$ recupera-se espontaneamente, entrando num compartimento de recuperados. Como em Sevá et al. 19, o modelo assume que a imunidade é temporária e que o animal torna-se novamente suscetível a uma taxa de $1 / 365$ dias. Um compartimento $R$ foi acrescido ao modelo $\mathrm{SEI}_{2} \mathrm{D}$, como ilustrado na Figura 1 em verde.

Todos os modelos foram implementados no programa R versão 3.2.3 (http://www.r-project.org) e resolvidos numericamente utilizando-se a biblioteca deSolve 30 .

\section{Calibração dos modelos para Araçatuba e Birigui}

Foram realizados inquéritos sorológicos caninos no período de setembro de 2015 a julho de 2016 nas áreas com maior incidência da doença humana nos municípios de Araçatuba e Birigui. Essa atividade foi realizada conjuntamente com os agentes de endemias dos Centros de Controle de Zoonoses (CCZ) desses dois municípios. Os inquéritos sorológicos, descritos em detalhes em Costa et al. 31, consistiram na identificação e coleta de amostra de sangue dos cães para teste da LVC (TR-DPP-Bio-Manguinhos e ELISA), usando-se o protocolo da rotina do programa de controle. As áreas selecionadas foram definidas com base em estudos anteriores que identificaram aglomerados de alto risco da infecção humana na área urbana dos municípios 4,16. As soroprevalências de LVC mensuradas nesses inquéritos foram de 8\% (IC95\%: 6-10) em Araçatuba 31 e 4\% (IC95\%: 3-5) em Birigui.

O tamanho da população canina em cada município $(N)$ foi estimado considerando-se a razão de um cão para cinco pessoas 32, totalizando 8.538 cães em Araçatuba e 11.958 em Birigui. Parâmetros de história de vida (taxa de natalidade e mortalidade dos cães, evolução da infecção por leishmaniose) foram obtidos na literatura (Tabela 1). Dados sobre o esforço de inquérito de cães, testagem e eutanásia foram disponibilizados pelo CCZ de cada município, referentes ao período de 2012 a 2014. Baseando-se nesses dados, a taxa de inquérito canino anual, $r$, foi calculada como a razão entre o número de cães avaliados e o número estimado de cães residentes no município. Usando valores dos três anos anteriores ao estudo, calculou-se uma taxa de inquérito de $9 \%$ em Araçatuba e $8 \%$ em Birigui. Já a cobertura anual de eutanásia foi calculada pela divisão do número de cães eutanásias e o número de cães com diagnóstico positivo, resultando em 43\% de eutanásia em Araçatuba e 66\% em Birigui. A rotina do programa de controle da LVC, dada a complexidade das atividades, não é feita de modo contínuo, isto é, nem sempre as atividades são realizadas com o mesmo esforço. Apesar disso, 
o modelo assume que o esforço é contínuo, sendo que as taxas anuais foram transformadas em taxas diárias de controle.

Os valores dos parâmetros são todos fixados em valores da literatura, exceto a taxa de transmissão $\beta$, que foi estimada com base na calibração dos modelos aos dados do estudo de prevalência. Para isso, a prevalência de acordo com o modelo foi calculada pela expressão:

$$
\text { Soroprevalência }\left(\text { modelo }{ }^{*}\right)=\left(I_{s}+I_{a}+D_{s}+D_{a}\right) / N \times 100
$$

em que o asterisco indica que os valores foram obtidos após o modelo ser calculado até o equilíbrio. Os valores de taxa de transmissão que resultam em uma soroprevalência igual ao observado nos inquéritos sorológicos foram: $\beta=3,06 \times 10^{-3} \mathrm{em}$ Araçatuba e $\beta=2,95 \times 10^{-3} \mathrm{em}$ Birigui.

A calibração do modelo com vetores necessitou um passo extra, pois foi necessário postular um tamanho populacional de flebotomíneos já que esta informação inexistia para as duas cidades. Como solução, optou-se por definir a população de vetores $\left(V_{1}+V_{2}+V_{3}\right)$ como a necessária para manter as mesmas taxas de transmissão $\beta$ mencionadas, encontradas no modelo sem vetores, assim como as mesmas soroprevalências. Encontrou-se como resultado que um tamanho populacional de aproximadamente 1,8 flebotomíneo por cão seria necessário para atender esses critérios (Tabela 1).

O modelo com recuperação de infectados foi calibrado aos dados de soroprevalência usando-se a mesma metodologia do modelo $\mathrm{SEI}_{2} \mathrm{D}$. Os valores encontrados estão descritos na Tabela 1.

\section{Cenários avaliados}

Os modelos calibrados levam em consideração os esforços de inquérito, diagnóstico e eutanásia realizados em Araçatuba e Birigui, conforme indicado na Tabela 1. Simulou-se, a partir daí, um cenário alternativo em que o controle é interrompido e no qual os parâmetros de inquérito foram igualados a 0. Observou-se o impacto da ausência do controle na soroprevalência em 10, 20, 30 e 40 anos e no equilíbrio. $\mathrm{O}$ aumento resultante da soroprevalência é utilizado para computar o efeito do controle atual:

$$
\text { efeito }=\frac{\text { Soroprevalência }}{\text { semcontrole }}
$$

Por fim, foi investigado um cenário de controle aumentado no qual o inquérito é aumentado para $25 \%$ dos cães anualmente (valor aproximadamente o triplo do que o empregado nos dois municípios). Da mesma forma, foi investigado o efeito na soroprevalência na mesma janela temporal.

\section{Cálculo do número reprodutivo}

A expressão para o número reprodutivo da LVC foi obtida para o modelo dinâmico sem vetores (modelo original) usando-se o método descrito em van den Driessche \& Watmough 33, apresentado no Material Suplementar (http://cadernos.ensp.fiocruz.br/site/public_site/arquivo/suppl -e00221418_1811.pdf). O objetivo foi verificar se o controle realizado atualmente nos municípios consegue reduzir o número reprodutivo abaixo de 1.

\section{Resultados}

Assumindo que a transmissão de LCV está no equilíbrio endêmico, foi estimado que a incidência diária de novas infecções em cães é de 0,65 caso em Araçatuba e 0,51 caso em Birigui (modelo SEI ${ }_{2} \mathrm{D}$ ). Esses valores sobem para 0,89 e 0,67, respectivamente, quando incluída a classe de recuperados no modelo ( $\left.\mathrm{SEI}_{2} \mathrm{DR}\right)$. A inclusão dos vetores tem pouco impacto na estimativa, sendo de 0,59 e 0,52 nas das cidades, respectivamente ( $\mathrm{SEI}_{2} \mathrm{DV}$ ). Em conjunto, os modelos indicam que, na presença das estratégias atuais de controle, uma infecção nova ocorre a cada 1 ou 2 dias. 
Figura 2

Soroprevalência de leishmaniose visceral canina (LVC) em Araçatuba, São Paulo, Brasil, em diferentes cenários de controle, baseado nos três modelos matemáticos descritos no texto.

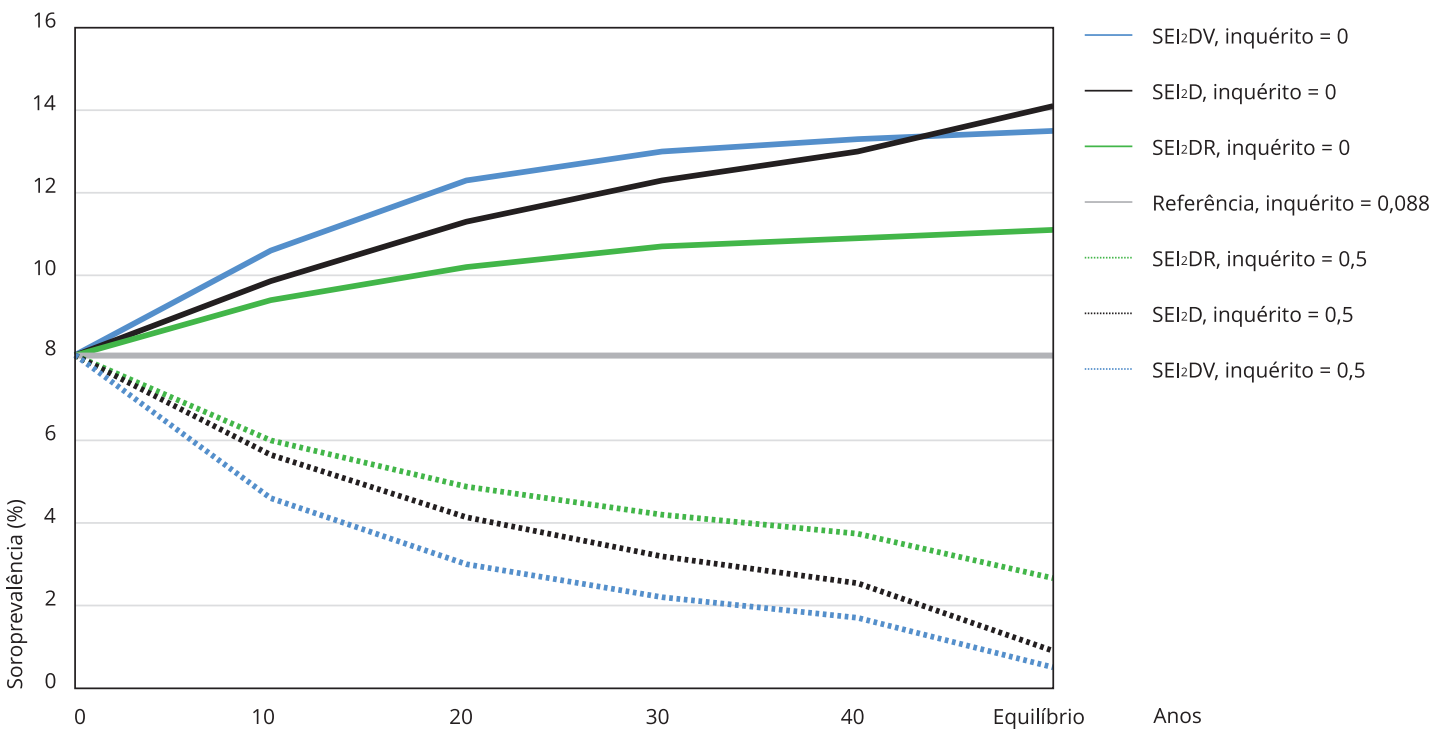

Nota: linhas sólidas indicam o efeito que ocorreria caso houvesse interrupção do controle; linhas pontilhadas simulam cenário de controle ampliado. 
Figura 3

Soroprevalência de leishmaniose visceral canina (LVC) em Birigui, São Paulo, Brasil, em diferentes cenários de controle utilizando eutanásia de cães soropositivos.

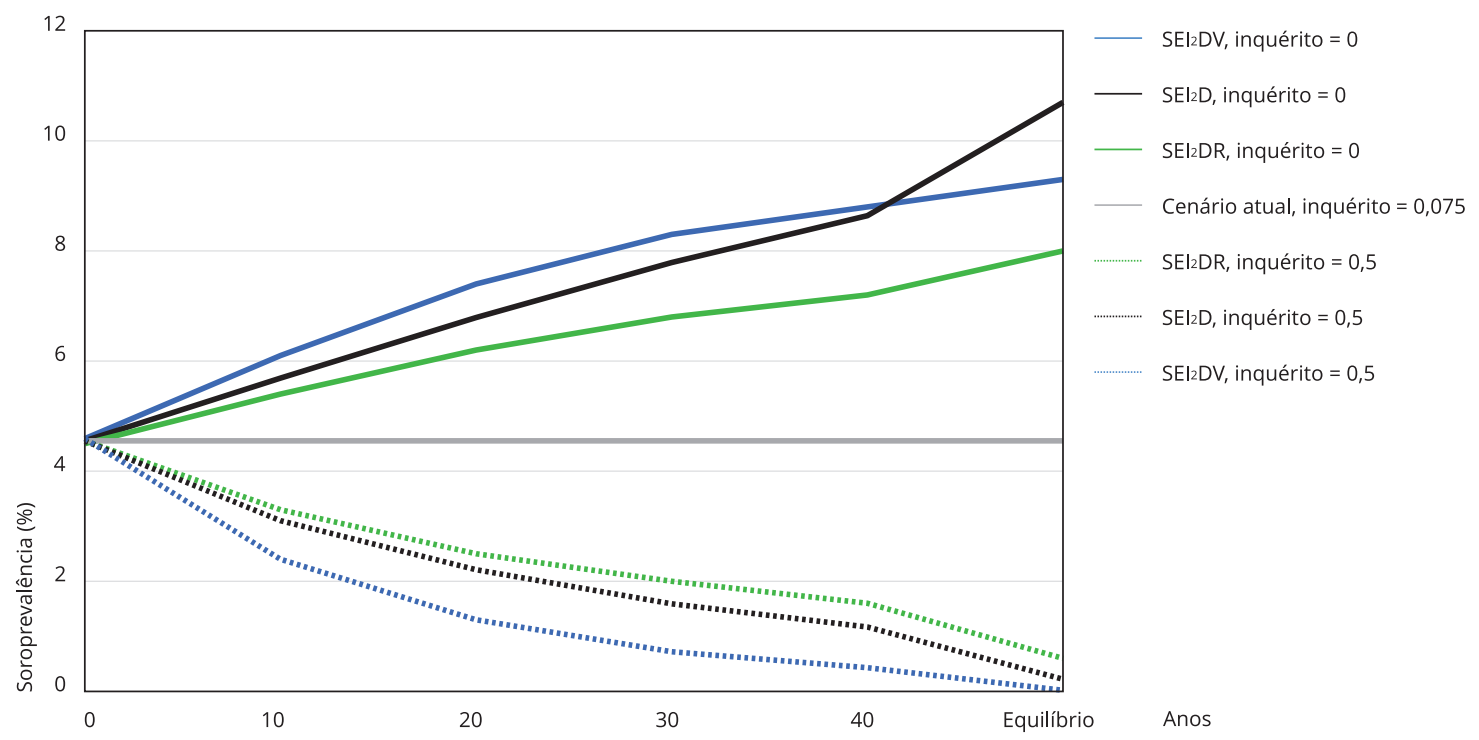

Nota: linhas sólidas indicam o efeito que ocorreria caso houvesse interrupção do controle; linhas pontilhadas simulam cenário de controle ampliado.

\section{Discussão}

Os resultados deste estudo mostraram que, em teoria, a eutanásia de cães soropositivos é capaz de controlar a infecção canina em cenários de inquérito contínuo de pelo menos $25 \%$ da população canina anualmente, seguido de eutanásia de cães soropositivos sintomáticos e assintomáticos com cobertura de pelo menos 50\%. Isso ocorreria mesmo em situações com reposição de 100\% dos cães eliminados por cães novos suscetíveis. $\mathrm{O}$ fato de ter sido obtido um valor de $\operatorname{Re}>1$, porém próximo da unidade, sugere que um esforço para reduzir em 10\% a transmissão poderia levar ao cenário de eliminação, de acordo com o modelo $\mathrm{SEI}_{2} \mathrm{D}$. Os modelos diferem quanto à janela de tempo necessária para alcançar esses limiares, sendo sempre de pelo menos 40 anos. O modelo com vetor mostrou-se o mais otimista, comparado com o modelo sem vetor. A inclusão de uma fração de recuperados implicou um esforço maior de inquérito e controle para atingir os mesmos resultados.

$\mathrm{O}$ esforço de inquérito realizado nas duas cidades, apesar de baixo em comparação com os valores teóricos ideais, mostrou-se capaz de reduzir em cerca de $20 \%$ a soroprevalência da LVC. Evidência do efeito do controle havia sido previamente identificado por Nunes et al. 34, em um estudo também realizado no Município de Araçatuba, onde verificaram correlação negativa entre a ocorrência de casos humanos de LV e a realização do controle do reservatório canino nos anos anteriores. Apesar dos desafios envolvidos na estratégia de controle por eutanásia de cães soropositivos, os autores observaram que com a interrupção do controle a incidência da infecção humana aumentou. Dessa forma, a redução de $20 \%$ na soroprevalência, quando comparada com um cenário sem controle, pode ter importância epidemiológica, uma vez que, pequenas alterações na dinâmica de transmissão canina possibilitam, em longo prazo, a diminuição de casos em humanos 4,19,20.

Atualmente, as estratégias de controle do reservatório canino da LV implementada no nível de saúde pública são o inquérito sorológico e a eutanásia de cães soropositivos, sendo que, até 2016, o PNVCLV preconizava a realização anual de inquérito sorológico canino censitário para regiões com transmissão intensa da doença ${ }^{35}$. Apesar de alcançar certo sucesso, as atividades de controle do reser- 
vatório canino atualmente implementadas não têm sido suficientes para interromper a transmissão da LVC 5,9. Na prática, a realização do controle por eutanásia enfrenta grande complexidade operacional, altos custos, e traz questões éticas e de ônus emocional para profissionais e tutores decorrentes das atividades de eutanásia, que fomentam a recusa na participação nos inquéritos e na posterior entrega de cães infectados 5,6,7,8. Nas duas cidades estudadas, que são bem estruturadas em comparação com a realidade brasileira, o controle de LVC realizado é bem menor do que o necessário para a eliminação, sendo que os motivos para as baixas coberturas de inquérito ( 7 a 8\%) estão entre os listados anteriormente. Para o alcance da meta da eliminação ou uma prevalência próxima a ela em 40 anos, as atividades de controle, segundo os resultados do presente estudo, teriam de ser pelo menos triplicadas.

Existem alguns pressupostos no estudo que precisam ser considerados. Os modelos assumem que apenas os cães verdadeiros soropositivos são eutanaseados. Contudo, os testes sorológicos utilizados na rotina têm especificidade $<100 \%$, o que resulta em uma probabilidade de ocorrência de diagnóstico falso-positivo e sacrifício desnecessário de cães não infectados 36,37. Na prática, isso implica que seria necessário realizar a eutanásia de uma quantidade maior de cães, de tal forma a alcançar as coberturas de eutanásia observadas no modelo. Considerando-se as dificuldades operacionais e de manutenção envolvidas na execução de inquérito sorológico, o aumento anual da cobertura dessa atividade seria o equivalente a triplicar o esforço atual em Araçatuba e em Birigui, o que parece ser pouco factível.

Uma solução apresentada por vários autores é a combinação ou substituição da eutanásia por outras estratégias de controle. Modelos dinâmicos da transmissão da LV têm sido utilizados para avaliar o efeito de diferentes medidas de controle, que, em geral, apontam para o uso de coleiras impregnadas com inseticida como a medida mais eficaz dentre as estratégias de eutanásia, tratamento e vacinação de cães 19,20,38. Sevá et al. 19 observaram que o encoleiramento de cães foi a estratégia mais eficaz, quando comparada com a eutanásia ou com a vacinação de cães, em um cenário no qual 90\% dos cães usavam a coleira e havendo reposição imediata das coleiras perdidas. No entanto, para que uma medida de controle possa ser utilizada no nível de saúde pública é necessário, além da comprovação de sua eficácia, que sua aplicação seja economicamente viável na rotina do programa, uma vez que os recursos são limitados.

Shimozako et al. 20 fizeram uma análise de custo-efetividade das medidas de controle considerando a dinâmica da infecção, e observaram que apesar dos desafios logísticos no uso de coleiras impregnadas com deltametrina, como a reposição das coleiras a cada seis meses ou precocemente devido a perdas, esta foi a estratégia de melhor custo-efetividade no controle da LV. Adicionalmente, estudos de campo também revelaram a efetividade dessa estratégia como medida capaz de promover a redução na prevalência da infecção canina, na taxa de flebotomíneos infectados e na incidência de casos humanos 11,12,14,15. Um estudo conduzido por Werneck et al. 15, no qual observou-se redução significativa da incidência de casos humanos a partir da associação de eutanásia e encoleiramento de cães, aponta como plausível um dos encontros do presente estudo, o de que a associação de estratégias poderia resultar em números reprodutivos menores do que a unidade e, consequentemente, na interrupção da transmissão.

A utilização de modelo dinâmico, apesar do uso de parâmetros baseados na literatura, implica que os resultados do estudo teórico são limitados aos casos em que o modelo é válido. E há muitas incertezas. Por exemplo, pouco se sabe sobre a capacidade vetorial de flebotomíneos infectados, o limiar de densidade vetorial por hospedeiro e a probabilidade de transmissão por meio das picadas em hospedeiros infectados. Esses são parâmetros essenciais para a calibração de modelos que consideram a dinâmica da infecção vetorial 19,20. Na prática, são atribuídos valores a esses parâmetros a fim de adequar o modelo à situação epidemiológica de LV. No presente estudo, foram verificadas semelhanças entre os resultados do modelo com as equações da dinâmica da infecção vetorial (modelo com vetor) e os do modelo que considera indiretamente a transmissão vetorial (modelo original) na avaliação da infecção canina. Portanto, dadas as incertezas quanto aos parâmetros vetoriais e prezando pela simplicidade matemática, o modelo original é o mais parcimonioso, ainda que possíveis variações na capacidade vetorial não sejam consideradas.

Os resultados alcançados pelos modelos de LVC foram bem sensíveis à introdução de um compartimento de recuperados. A presença de 10\% de recuperação implica que seja necessária uma força de infecção mais alta para manter os mesmos níveis de soroprevalência. Com uma transmissão maior, é necessário um esforço maior de controle para reduzir a soroprevalência, isto explica o menor impacto 
do controle nesse modelo em relação aos outros. Na literatura, os modelos de LVC variam em relação à modelagem do compartimento de recuperados, e isto resulta da lacuna de estudos sobre o tema. Os melhores estudos existentes são coortes (Oliva et al. ${ }^{39}$, por exemplo), que poderiam ser replicadas com tamanhos amostrais maiores e seguimentos também maiores. Os dados resultantes trariam melhoras significativas para as estimativas de custo-efetividade de estratégias de controle de LVC.

Outra limitação dos modelos é o pressuposto de que todos os cães estão expostos de forma homogênea ao risco de contato com o vetor. Porém, como observado por Costa et al. 31, o risco varia espacialmente, dependendo das características dos cães, de seus tutores e dos domicílios. Esses autores verificaram a existência de um padrão local da transmissão da infecção no entorno dos domicílios, relacionado com as características do vetor, e observaram uma associação positiva entre a infecção e o local onde os cães são mantidos ao longo do dia.

Os modelos não consideram outros hospedeiros em potencial, como animais silvestres, que poderiam reduzir o impacto do controle caso estivessem presentes. Finalmente, o controle é implementado continuamente com o mesmo esforço, o que não ocorre em situações reais, em que o controle é descontinuado devido a problemas orçamentários e operacionais. Dessa forma, os resultados deste estudo devem ser avaliados com cautela e de um ponto de vista realista, observando que, apesar das dificuldades associadas à eutanásia de cães soropositivos, esta é uma medida que tem contribuído para a diminuição da prevalência canina e que, no momento, é a estratégia utilizada no nível de saúde pública enquanto não se viabiliza a aplicação de outras medidas de controle. Mudanças nesse cenário poderão e deverão ocorrer na medida em que novas medidas de controle, associadas ou não à eutanásia, apresentem melhor custo-efetividade do que a estratégia atual.

\section{Colaboradores}

D. N. C. C. Costa contribuiu com a concepção e planejamento do estudo, coleta de dados de campo, coordenação e supervisão da coleta de dados de campo, suporte ao gerenciamento de dados, supervisão dos ensaios laboratoriais, análise de dados, coordenação da pesquisa e elaboração do manuscrito. C. T. Codeço contribuiu com a concepção e planejamento do estudo, coordenação da pesquisa, análise de dados, elaboração do manuscrito, leitura crítica e contribuição para a elaboração da versão final deste artigo. P. M. M. Bermudi contribuiu com a coleta de dados de campo, suporte ao gerenciamento de dados, leitura crítica e contribuição para a elaboração da versão final deste manuscrito. L. A. C. Rodas e C. M. Nunes contribuíram com a concepção e planejamento do estudo, coleta de dados de campo, suporte ao gerenciamento de dados, leitura crítica e contribuição para a elaboração da versão final deste manuscrito. R. M. Hiramoto e J. E. Tolezano contribuíram com a concepção e planejamento do estudo, supervisão dos ensaios laboratoriais, leitura crítica e contribuição para a elaboração da versão final deste manuscrito. F. Chiaravalloti Neto contribuiu com a concepção e planejamento do estudo, coordenação da pesquisa, elaboração do manuscrito, leitura crítica e contribuição para a elaboração da versão final deste artigo.

\section{Informações adicionais}

ORCID: Danielle Nunes Carneiro Castro Costa (0000-0002-9264-2937); Cláudia Torres Codeço (0000-0003-1174-178X); Patricia Marques Moralejo Bermudi (0000-0002-5825-6389); Lilian Aparecida Colebrusco Rodas (0000-0003-4840-9588); Cáris Maroni Nunes (0000-0002-5463-3845); Roberto Mitsuyoshi Hiramoto (0000-0002-74041505); José Eduardo Tolezano (0000-0003-30550508); Francisco Chiaravalloti Neto (0000-00032686-8740).

\section{Agradecimentos}

Agradecemos a Flávio Codeço Coelho por ter elaborado a fórmula do número de reprodução efetivo e a Gustavo Paganini Canal por simplificar esta fórmula. 


\section{Referências}

1. Coordenação-Geral de Desenvolvimento da Epidemiologia em Serviços, Secretaria de Vigilância em Saúde, Ministério da Saúde. Guia de vigilância em saúde. Brasília: Ministério da Saúde; 2016.

2. Bianchi MV, Fredo G, Tagliari NJ, Leite Filho RV, De Lorenzo C, Lupion CG, et al. Leishmaniose visceral canina autóctone na região urbana de Porto Alegre, RS, Brasil. Acta Sci Vet 2016; 44 Suppl 1:110.

3. Oliveira AM, Vieira CP, Dibo MR, Guirado MM, Rodas LAC, Chiaravalloti Neto F. Dispersal of Lutzomyia longipalpis and expansion of canine and human visceral leishmaniasis in São Paulo State, Brazil. Acta Trop 2016; 164:233-42.

4. Costa DNCC, Bermudi PMM, Rodas LAC, Nunes CM, Hiramoto RM, Tolezano JE, et al. Leishmaniose visceral em humanos e relação com medidas de controle vetorial e canino. Rev Saúde Pública 2018; 52:92.

5. von Zuben APB, Donalísio MR. Dificuldades na execução das diretrizes do Programa de Vigilância e Controle da Leishmaniose Visceral em grandes municípios brasileiros. Cad Saúde Pública 2016; 32:e00087415.

6. Romero GA, Boelaert M. Control of visceral leishmaniasis in Latin America: a systematic review. PLoS Negl Trop Dis 2010; 4:e584.

7. Machado CJS, Silva EG, Vilani RM. O uso de um instrumento de política de saúde pública controverso: a eutanásia de cães contaminados por leishmaniose no Brasil. Saúde Soc 2016; 25:247-58

8. Silva STP, Marqués LFV, Lamounier KCC, Castro JM, Borja-Cabrera GP. Leishmaniose visceral humana: reflexões éticas e jurídicas acerca do controle do reservatório canino no Brasil. Rev Bioét Derecho 2017; (39):135-51.

9. Werneck GL. Controle da leishmaniose visceral no Brasil: o fim de um ciclo? Cad Saúde Pública 2016; 32:eED010616.

10. Nery G, Becerra DRD, Borja LS, MagalhãesJunior JT, Souza BMPS, Franke CR, et al. Avaliação da infectividade parasitária a Lutzomyia longipalpis por xenodiagnóstico em cães tratados para leishmaniose visceral naturalmente adquirida. Pesq Vet Bras 2017; 37:701-7.

11. Andrade AJD, Quint BB, Raffoul GES, Werneck GL, Rangel EF, Romero GAS. Effectiveness of dog collars impregnated with $4 \%$ deltamethrin in controlling visceral leishmaniasis in Lutzomyia longipalpis (Diptera: Psychodidade: Phlebotominae) populations. Mem Inst Oswaldo Cruz 2018; 113:e170377.

12. Kazimoto TA, Amora SSA, Figueiredo FB, Magalhães JME, Freitas YBN, Sousa MLR, et al. Impact of $4 \%$ deltamethrin-impregnated dog collars on the prevalence and incidence of canine visceral leishmaniasis. Vector Borne Zoonotic Dis. 2018; 18:356-63.
13. Toepp A, Larson M, Wilson G, Bennett C, Leal-Lima A, Anderson B, et al. Randomized, controlled, double-blinded field trial to assess Leishmania vaccine effectiveness as immunotherapy for canine leishmaniosis. Vaccine 2018; 36:6433-41.

14. Maroli M, Mizzon V, Siragusa C, D’Oorazi A, Gradoni L. Evidence for an impact on the incidence of canine leishmaniasis by the mass use of deltamethrin-impregnated dog collars in southern Italy. Med Vet Entomol 2001; 15:358-63

15. Werneck GL, Figueiredo F, Cruz, MP. Impact of $4 \%$ deltamethrin-impregnated dog collars on the incidence of human visceral leishmaniasis. Int J Infect Dis 2018; 73:42.

16. Vieira CP, Oliveira AM, Rodas LAC, Dibo MR, Guirado MM, Chiaravalloti Neto F. Temporal, spatial and spatiotemporal analysis of the occurrence of visceral leishmaniasis in humans in the City of Birigui, State of São Paulo, from 1999 to 2012. Rev Soc Bras Med Trop 2014 47:350-8.

17. Burattini MN, Coutinho FAB, Lopez LF, Massad E. Modelling the dynamic of leishmaniasis considering human, animal host and vector population. J Biol Syst 1998; 6:337-56.

18. Rosales JC, Yang HM. Modelo matemático para descrever transmissão de leishmaniose. TEMA - Trends in Applied and Computational Mathematics 2006; 7:337-46.

19. Sevá AP, Ovallos FG, Amaku M, Carrillo E, Moreno J, Galati EA, et al. Canine-based strategies for prevention and control of visceral leishmaniasis in Brazil. PLoS One 2016; 11:e0160058.

20. Shimozako HJ, Wu J, Massad E. The preventive control of zoonotic visceral leishmaniasis: efficacy and economic evaluation. Comput Math Methods Med 2017; 2017:4797051.

21. Lanotte G, Rioux JA, Perieres J, Vollhardt Y. Ecology of leishmaniasis in the south of France. 10. Developmental stages and clinical characterization of canine leishmaniasis in relation to epidemiology. Ann Parasitol Hum Comp 1979; 54:277-295.

22. Fisa R, Gállego M, Castillejo S, Aisa MJ, Serra T, Riera MC, et al. Epidemiology of canine leishmaniosis in Catalonia (Spain): the example of the Priorat focus. Vet Parasitol 1999; 83:87-97.

23. Moreno J, Alvar J. Canine leishmaniasis: epidemiological risk and the experimental model. Trends Parasitol 2002; 18:399-405.

24. Costa DNCC, Codeço CT, Silva MA, Werneck GL. Culling dogs in scenarios of imperfect control: realistic impact on the prevalence of canine visceral leishmaniasis. PLoS Negl Trop Dis 2013; 7:e2355. 
25. Nunes CM, Lima VMF, Paula HB, Perri SHV, Andrade AM, Dias FEF, et al. Dog culling and replacement in an area endemic for visceral leishmaniasis in Brazil. Vet Parasitol 2008; 153:19-23.

26. Maia C, Nunes M, Cristóvão J, Campino L. Experimental canine leishmaniasis: clinical, parasitological and serological follow-up. Acta Trop 2010; 116:193-9.

27. Pozio E, Grandote L, Betti S, Granita M. Leishmaniasis in Toscana (Italy): VI. Canine leishmaniasis in the focus of Monte Argentário (Grosseto). Acta Trop 1981; 38:383-93.

28. Marzochi MCA, Coutinho SG, Sabroza PC. Leishmaniose visceral canina no Rio de Janeiro - Brasil. Cad Saúde Pública 1985; 1:432-46.

29. Nishiura H. Mathematical and statistical analyses of the spread of dengue. Dengue Bulletin 2006; 30:51-67.

30. Soetaert K, Petzoldt T, Setzer RW. Solving differential equations in R: package deSolve. J Stat Soft 2010; 33:1-25.

31. Costa DNCC, Blangiardo M, Rodas LAC, Nunes CM, Hiramoto RM, Tolezano JE, et al. Canine visceral leishmaniasis in Araçatuba, state of São Paulo, Brazil, and its relationship with characteristics of dogs and their owners: a cross-sectional and spatial analysis using a geostatistical approach. BMC Vet Res 2018; 14:229.

32. Nunes CM, Martines DA, Fikaris S, Queiroz LH. Avaliação da população canina da zona urbana do Município de Araçatuba, São Paulo. Rev Saúde Pública 1997; 31:308-9.

33. van den Driessche P, Watmough J. Reproduction numbers and sub-threshold endemic equilibria for compartmental models of disease transmission. Math Biosci 2002; 180:2948.
34. Nunes CM, Pires MM, da Silva KM, Assis FD, Gonçalves Filho J, Perri SH. Relationship between dog culling and incidence of human visceral leishmaniasis in an endemic area. Vet Parasitol 2010; 170:131-3.

35. Ministério da Saúde. Manual de vigilância e controle da leishmaniose visceral. Brasília: Ministério da Saúde; 2014.

36. Laurenti MD, Rossi CN, da Matta VLR, Tomokane TY, Corbett CEP, Secundino NFC, et al Asymptomatic dogs are highly competent to transmit Leishmania (Leishmania) infantum chagasi to the natural vector. Vet Parasitol 2013; 196:296-300.

37. Grimaldi Jr. G, Teva A, Ferreira AL, dos Santos CB, Pinto Id, de-Azevedo CT, et al. Evaluation of a novel chromatographic immunoassay based on Dual-Path Platform technology (DPP1 CVL rapid test) for the serodiagnosis of canine visceral leishmaniasis. Trans R Soc Trop Med Hyg 2012; 106:54-9.

38. Dye C. The logic of visceral leishmaniasis control. Am J Trop Med Hyg 1996, 55:125-30.

39. Oliva G, Scalone A, Foglia-Manzillo V, Gramiccia M, Pagano A, Di-Muccio T, et al. Incidence and time course of Leishmania infantum infections examined by parasitological, serologic, and nested-PCR techniques in a cohort of naive dogs exposed to three consecutive transmission seasons. J Clin Microbiol 2006; 44:1318-22.

32. Courtenay O, Peters NC, Rogers ME, Bern C. Combining epidemiology with basic biology of sand flies, parasites, and hosts to inform leishmaniasis transmission dynamics and control. PLoS Pathog 2017; 13:e1006571. 


\section{Abstract}

Visceral leishmaniasis is an emerging and neglected disease that is currently expanding to urban areas. The incidence of human disease is related to canine infection. Araçatuba and Birigui are municipalities (counties) in the state of São Paulo, Brazil, with 8-10\% seroprevalence of canine infection and that employ control strategies targeting the canine reservoir, based on serological survey and culling of seropositive dogs. Using data from these control programs to parameterize mathematical models, this study assessed the efficacy of these activities. We estimated that current control is capable of reducing the incidence of canine visceral leishmaniasis (CVL) by approximately $20 \%$. Assuming continuous control and three times the current serological survey activities in Araçatuba and Birigui, culling dogs with a positive CVL diagnosis would be effective for the control of canine infection. Although theoretically possible, in practice the control of CVL with the currently recommended strategies is insufficient, since it would require overcoming the difficulties in these activities, such as lack of material, human, and financial resources, besides associated ethical and legal issues.

Leishmania; Visceral Leishmaniasis; Dog Diseases; Disease Reservoirs

\section{Resumen}

La leishmaniasis visceral es una enfermedad emergente y pasada por alto en proceso de expansión hacia áreas urbanas. La incidencia de la enfermedad humana está relacionada con la infección canina. Araçatuba y Birigui son municipios del estado de São Paulo, Brasil, con una seroprevalencia de infección canina de un 8 a un $10 \%$ que emplean estrategias de control dirigidas al reservorio canino, basado en una encuesta serológica y eutanasia de los perros seropositivos. Usando datos de esos programas de control para proporcionar parámetros en modelos matemáticos, este estudio evaluó la eficacia de esas actividades. Estimamos que el control actualmente empleado es capaz de reducir cerca de un 20\% la incidencia de casos de leishmaniasis visceral canina (LVC). Si se considera un control continuo y un esfuerzo de las actividades de encuesta serológica igual al triple de la media de lo observado en Araçatuba y Birigui, la actividad de eutanasia de perros con diagnóstico positivo sería efectiva para el control de la infección canina. A pesar de que teóricamente es posible, en la práctica el control de la LVC con las estrategias preconizadas actualmente es insuficiente, puesto que exigiría superar dificultades a las que se enfrentan estas actividades como la falta de recursos materiales, humanos y financieros, además de las cuestiones éticas y jurídicas asociadas.

Leishmania; Leishmaniasis Visceral;

Enfermedades de los Perros; Reservorios de Enfermedades
Recebido em 21/Nov/2018

Versão final reapresentada em 28/Jun/2019

Aprovado em 14/Ago/2019 